\title{
Inibidores da enzima de conversão da angiotensina ou antagonistas dos recetores da angiotensina: evidências na mortalidade $e$ eventos cardiovasculares major em diabéticos hipertensos
}

Paula Mendes, ${ }^{1}$ Vítor Portela Cardoso ${ }^{2}$

\section{RESUMO}

Objetivo: Rever a literatura, graduando a evidência do efeito dos inibidores da enzima de conversão da angiotensina (IECA) ou dos antagonistas dos receptores da angiotensina (ARA) na mortalidade ou ocorrência de eventos cardiovasculares major nos diabéticos hipertensos. Fontes de Dados: National Guideline Clearinghouse, Guidelines Finder, Canadian Medical Association Practice Guidelines, Cochrane, DARE, Bandolier, MEDLINE, Índex de Revistas Médicas Portuguesas e Direção-Geral da Saúde.

Métodos: Os dois autores realizaram, de forma independente, uma pesquisa de meta-análises (MA), revisões sistemáticas (RS), ensaios clínicos aleatorizados e controlados (ECAC) e normas de orientação clínica (NOC), publicados entre 31/08/2009 e 31/08/2014, nas línguas portuguesa, espanhola, inglesa e francesa, utilizando os termos MeSH: Diabetes Mellitus AND Hypertension AND Angiotensin-Converting Enzyme Inhibitors OR Angiotensin Receptor Antagonists. Foi utilizada a escala Strength Of Recommendation Taxonomy para atribuição dos níveis de evidência (NE) e forças de recomendação (FR).

Resultados: Dos 478 artigos obtidos, foram incluídas 5 MA e 6 NOC. A MA da Cochrane demonstrou que os IECA diminuíam significativamente a mortalidade por todas as causas (NE 1) e a MA de Nakao concluiu que esta classe diminuía o risco de enfarte do miocárdio (NE 1). Já o uso de ARA demonstrou diminuir o risco de AVC na MA de Savarese. A MA de Wu não demonstrou diferenças significativas entre as duas classes. Recentemente, a MA de Cheng verificou que os IECA diminuíam significativamente a mortalidade por todas as causas, a mortalidade cardiovascular, o enfarte do miocárdio e a insuficiência cardíaca (NE 1). Nesta MA, os ARA diminuíram o risco de insuficiência cardíaca. A maioria das NOC incluídas recomendava os IECA ou ARA como primeira linha.

Conclusões: Nos diabéticos, os efeitos dos IECA e ARA parecem ser diferentes relativamente à mortalidade e eventos cardiovasculares. A evidência encontrada foi robusta e consistentemente favoreceu o uso de IECA como primeira opção na prevenção da morbimortalidade nesta população (FR A).

Palavras-chave (DeCS): Diabetes mellitus; Hipertensão arterial; Inibidores da enzima conversora da angiotensina; Antagonistas de receptores da angiotensina.

\section{INTRODUÇÃO}

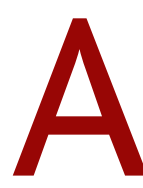

hipertensão arterial (HTA) é uma comorbilidade comum da diabetes mellitus (DM), ${ }^{1-2}$ estimando-se que até $70,9 \%$ dos indivíduos diabéticos em Portugal sejam hipertensos. ${ }^{3}$ Estas duas patologias são fatores de risco independentes e aditivos para doença cardiovascular (DCV)..$^{1-2,4}$ Esta é a principal causa de morbi-mortalidade em pessoas com DM, sendo responsável por $50 \%$ ou mais de todas as mortes. ${ }^{1}$ Foi demonstrado que o controlo dos fatores de risco cardiovasculares era eficaz na prevenção ou no retardar da DCV nestes indivíduos. ${ }^{1}$

${ }^{1}$ Médica Assistente de Medicina Geral e Familiar. ARS Norte

2Médico Assistente de Medicina Geral e Familiar. UCSP Esporões, ACES Cávado I Braga 
Existe uma vasta variedade de fármacos anti-hipertensores. As últimas recomendações da European Society of Hypertension/European Society of Cardiology (ESH/ESC) afirmam que os principais benefícios da terapêutica anti-hipertensora se devem à diminuição da pressão arterial per se e são, em grande parte, independentes do fármaco utilizado. ${ }^{4}$

O sistema renina-angiotensina-aldosterona (SRAA) é um importante regulador cardiovascular e renal, sendo que a sua desregulação perpetua uma cascata pró-inflamatória, pró-trombótica e aterogénica, que está na base das lesões de órgão-alvo. ${ }^{5}$ Algumas meta-análises (MA) demonstraram que a supressão deste sistema reduzia a mortalidade cardiovascular nos indivíduos hipertensos com ou sem DM. ${ }^{6-7}$

Os inibidores da enzima de conversão da angiotensina (IECA) e os antagonistas dos recetores da angiotensina (ARA) são os principais fármacos utilizados no bloqueio do SRAA, através de diferentes mecanismos de ação. ${ }^{5,8}$ Os IECA inibem a enzima que converte a angiotensina (ANG) I em II (substância vasoconstritora), mas não bloqueiam toda a produção de ANG II. Além da via clássica, $40 \%$ da ANG II é produzida por uma via alternativa, o chamado "fenómeno de escape". Por sua vez, os ARA atuam no final da cascata do SRAA, inibindo a ligação da ANG II ao recetor AT1. Uma vez que estas duas classes (IECA e ARA) atuam em diferentes locais do SRAA, questionou-se se os resultados relativos ao risco de morte ou eventos cardiovasculares seriam também diferentes. ${ }^{5} \mathrm{O}$ estudo HOPE (de 2000), demonstrou uma diminuição de eventos cardiovasculares com o ramipril. ${ }^{9}$ Mais tarde, o ensaio clínico ROADMAP (de 2011) demonstrou que no grupo tratado com olmesartan houve um maior número de eventos cardiovasculares fatais comparativamente com o placebo (15 vs $3, p=0,01) .^{10}$ No mesmo ano foi publicado outro ensaio clínico (ORIENT), cujos resultados mostraram que o uso de olmesartan se associava ao aumento do risco de mortalidade cardiovascular nos diabéticos com nefropatia. ${ }^{11}$

Perante estes resultados, a United States Food and Drug Administration (FDA) realizou uma revisão de segurança. No último comunicado em 2014, esta instituição concluía que não havia uma evidência clara para o aumento de risco cardiovascular com o uso de olmesartan em diabéticos hipertensos, não alterando as recomendações para o uso deste fármaco. ${ }^{12}$

Inicialmente, os ARA foram apontados como sendo mais benéficos que os IECA, uma vez que o mecanismo de ação dos ARA não se associa a "fenómenos de escape" de produção de ANG II. ${ }^{13}$ No entanto, alguns estudos sugeriram que a hiperestimulação que os ARA exercem nos recetores AT1 não seria assim tão benéfica, podendo mesmo causar hipertrofia cardíaca, fibrose vascular e diminuição da neovascularização. ${ }^{14} \mathrm{Além}$ disso, uma possível explicação para o benefício cardioprotetor dos IECA, relativamente aos ARA, presume-se que se possa dever ao facto dos IECA também atuarem no sistema das bradicininas. Ao inibirem a conversão da bradicinina em peptídeos inativos, o aumento da bradicinina exerce efeitos vasodilatadores e de inibição da agregação plaquetária. ${ }^{14}$

Apesar dos diversos resultados obtidos nos estudos, 0 padrão de prescrição de anti-hipertensores em Portugal revelou um crescimento de $54 \%$ na prescrição de ARA de 2000 para 2011. De facto, em 2011, a prescrição de IECA e ARA em monoterapia era sobreponível, respetivamente, $10,3 \%$ e 9,8\%. Já nos regimes isolados ou em associação, a prescrição de ARA em Portugal era superior (31\%) relativamente a Inglaterra $(11 \%) .^{15}$

O objetivo deste trabalho foi rever a evidência do efeito dos IECA ou ARA nos indivíduos diabéticos hipertensos quanto à mortalidade por todas as causas, mortalidade cardiovascular ou ocorrência de eventos cardiovasculares major.

\section{MÉTODOS}

Foi realizada uma pesquisa de MA, revisões sistemáticas (RS), ensaios clínicos aleatorizados e controlados (ECAC) e normas de orientação clínica (NOC) nas seguintes fontes de dados: National Guideline Clearinghouse, Guidelines Finder, Canadian Medical Association Practice Guidelines, Cochrane, DARE, Bandolier, MEDLINE, Direção-Geral da Saúde (DGS) e Índex de Revistas Médicas Portuguesas. Para isso foram utilizados os termos MeSH: Diabetes Mellitus AND Hypertension AND Angiotensin-Converting Enzyme Inhibitors OR Angiotensin Receptor Antagonists e os termos DeCS correspondentes: Diabetes Mellitus e Hipertensão e Inibidores da enzima conversora da angiotensina ou Antagonistas de receptores da angiotensina. Incluíram-se os artigos publicados entre 31 de agosto de 2009 e 31 de agosto de 2014 nas línguas portuguesa, inglesa, francesa e espanhola.

Os dois autores realizaram a seleção dos artigos de forma independente e oculta um do outro. No caso de opiniões divergentes à inclusão/ exclusão de um artigo, os autores decidiram em consenso após discussão argumenta- 
tiva. A avaliação final da qualidade e nível de evidência dos artigos incluídos foi realizada conjuntamente.

Foram incluídos os artigos cuja população fosse constituída por indivíduos diabéticos hipertensos com idade igual ou superior a 18 anos, sendo a intervenção o uso de IECA ou ARA em monoterapia (independentemente do tipo ou da dose) comparativamente com placebo, ARA ou IECA ou ainda outro anti-hipertensor. Os resultados a avaliar foram a mortalidade por todas as causas, a mortalidade cérebro-cardiovascular e os eventos cardiovasculares major, nomeadamente enfarte agudo do miocárdio (EAM), acidente vascular cerebral (AVC) e insuficiência cardíaca (IC).

Foram usados como critérios de exclusão: artigos duplicados, estudos observacionais e estudos que incluíssem grávidas ou indivíduos com doença renal terminal, uma vez que estes apresentam taxas de eventos cardiovasculares superiores aos restantes diabéticos, além de que têm respostas diferentes aos anti-hipertensores.

Não foram, ainda, incluídos estudos dirigidos à avaliação da relação custo-efetividade, efeitos laterais dos fármacos, nem a qualidade de vida dos indivíduos.

Foi adotada a escala Strengh of Recommendation Taxonomy (SORT), da American Academy of Family Physicians, para a avaliação dos níveis de evidência (NE) e atribuição de forças de recomendação (FR). ${ }^{16}$

\section{RESULTADOS}

Obteve-se um total de 478 referências, das quais foram selecionadas 11: cinco MA e seis NOC. O fluxograma da seleção dos artigos está representado na Figura 1.

A MA de Cheng e colaboradores (2014) incluiu 35 ECAC num total de 56.444 indivíduos com diabetes maioritariamente hipertensos (Quadro I). ${ }^{17}$ Os autores concluíram que os IECA diminuíam significativamente o risco de mortalidade por todas as causas em $13 \%$ (RR $=0,87$; IC95\% 0,78-0,98), o risco de mortalidade cardiovascular em $17 \%$ $(\mathrm{RR}=0,83$; IC95\% 0,70-0,99) e os eventos cardiovasculares em 14\% (RR=0,86; IC95\% 0,77-0,95), nomeadamente EAM em $21 \%$ e IC em 19\%, comparativamente com o placebo, sem tratamento ou outro anti-hipertensor. Por sua vez, os ARA não demonstraram reduzir significativamente a mortalidade por todas as causas nem a mortalidade cardiovascular. Relativamente aos eventos cardiovasculares $m a$ jor, os ARA apenas diminuíram significativamente o risco de IC em 30\% (RR=0,70; IC95\% 0,59-0,82). Nenhuma das classes diminuiu o risco de AVC. Os autores desta MA concluíram que os IECA deviam ser considerados como primeira linha para prevenção da morbi-mortalidade nos indivíduos com DM. Os autores desta MA avaliaram a qualidade dos estudos incluídos, tendo $60 \%$ pontuado mais de três pontos na escala de Jadad. Pela boa qualidade dos estudos incluídos e por estarem orientados para o paciente foi atribuído a esta MA um NE 1.

Em 2011, a MA de Wu e colaboradores, que estudou diabéticos hipertensos, não demonstrou resultados significativos na redução da mortalidade por todas as causas com o uso de IECA, comparativamente com os ARA (Quadro I). ${ }^{18}$ Foram também estudados os efeitos dos bloqueadores dos canais do cálcio (BCC), bloqueadores beta adrenérgicos (BB) e diuréticos. Wu e colaboradores categorizaram os anti-hipertensores pela probabilidade de melhor opção terapêutica na redução da mortalidade por todas as causas. Assim, em primeiro lugar, colocaram a associação IECA com BCC, seguida da associação IECA com diurético, em terceiro lugar IECA em monoterapia e em sexto lugar ARA. Os autores realizaram a MA através do modelo Bayesiano, que combinou a comparação direta e indireta dos estudos, estimando assim os efeitos relativos entre os anti-hipertensores estudados. Os autores desta MA concluíram que tendo em conta os custos, os IECA deviam ser usados como primeira linha no tratamento da HTA em diabéticos. Foi atribuído à MA de Wu um NE 1.

Nakao e colaboradores publicaram, em 2011, uma MA que estudou 19 ECAC em indivíduos com diabetes maioritariamente hipertensos. Os resultados demonstraram que o bloqueio do SRAA diminuía significativamente o risco de eventos cardiovasculares major e EAM, comparativamente com o placebo ou outros anti-hipertensores (Quadro I). ${ }^{19} \mathrm{Além}$ disso, verificaram uma tendência para a diminuição do risco do resultado composto MACE (mortalidade CV, EAM e AVC não fatal). Especificando por classe terapêutica, Nakao e colaboradores concluíram que apenas os IECA diminuíam o risco de EAM e mostraram uma tendência para diminuir o MACE. Adicionalmente, os resultados obtidos quanto à ausência de benefício com os ARA não se modificavam quando eram excluídos os ECAC, como o ROADMAP. Todos os estudos foram classificados com qualidade suficiente pela escala Agency for Healthcare Research and Quality for Sistematic Reviews. Foi atribuído à MA de Nakao um NE 1.

A quarta MA incluída teve a chancela da Cochrane e foi publicada em 2012. Incluiu $22 \mathrm{ECAC}$, em indivíduos com diabetes maioritariamente com HTA (Quadro I) ${ }^{20}$ Os IECA 
diminuíram significativamente o risco de mortalidade por todas as causas quando comparados com o placebo ou sem tratamento. Em oposição, não houve diminuição na mortalidade por todas as causas com os ARA, comparativamente com placebo/sem tratamento. Apesar da aparente superioridade dos IECA, nos cinco estudos em que houve comparação direta com os ARA não foram encontradas diferenças significativas na mortalidade por todas as causas. Os autores desta MA concluíram que os IECA podiam ser rotineiramente considerados para a prevenção primária da mortalidade em diabéticos, independentemente dos níveis de pressão arterial,

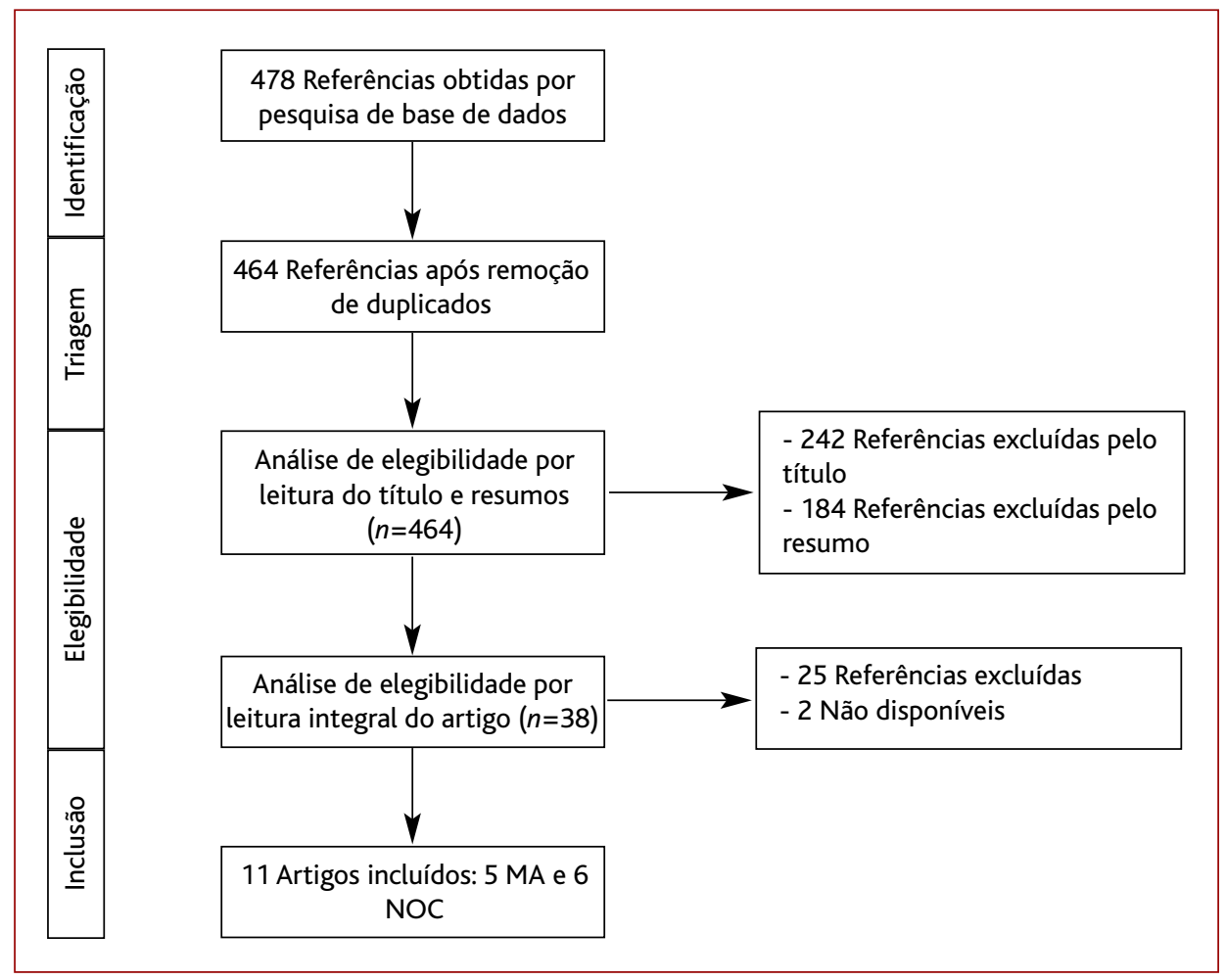

Figura 1. Fluxograma de seleção dos artigos

embora fossem necessários mais estudos para avaliar a relação custo-efetividade desta abordagem. Foi atribuído à MA da Cochrane um NE 1.

Savarese e colaboradores publicaram, em 2013, a sua MA onde analisaram 26 ECAC. Destes, os autores da presente revisão apenas optaram por incluir os ECAC relativos aos ARA, uma vez que os ECAC que estudaram os IECA tinham sido maioritariamente realizados em indivíduos sem DM (Quadro I). ${ }^{21}$ Esta MA obteve um resultado singular, associando o uso de ARA a uma diminuição significativa do risco de AVC em 9,1\% (RR=0,70; IC95\% 0,59-0,82) e do resultado composto (mortalidade CV, EAM e AVC) em 7\% (RR 0,92; IC95\% 0,869-0,975). Concluíram o seu trabalho afirmando que os ARA eram uma opção válida para a redução da morbi-mortalidade quando os IECA não pudessem ser usados. Foi atribuído a esta MA um NE 1.

Foram ainda incluídas seis NOC (Quadro II). Todas eram baseadas na evidência, sendo que a ESH/ESC era também suportada por opinião de peritos. No geral, nenhuma dá preferência a uma única classe farmacológica no tratamento da HTA em diabéticos.

A ESH/ESC, emitida em 2013, refere que todas as classes anti-hipertensoras podem ser usadas em doentes com diabetes, devendo-se individualizar a prescrição conforme as comorbilidades dos pacientes. ${ }^{4}$ Acrescenta ainda que os bloqueadores do SRAA podem ser preferidos, especialmente na presença de microalbuminúria ou proteinúria. Estas recomendações basearam-se numa revisão clássica de $2007^{22}$ e numa RS de $2005 .^{23}$

A mais recente Eight Joint National Committee (JNC 8) também recomenda o uso de um diurético tiazídico, BCC, IECA ou ARA, na população em geral, incluindo indivíduos diabéticos. Nos indivíduos de raça negra com DM, o tratamento anti-hipertensivo inicial recomendado deverá incluir um diurético tiazídico ou um BCC. ${ }^{24}$ Para emitir estas recomendações, os autores apenas incluíram ECAC que comparassem diferentes classes farmacológicas frente-a-frente. Optaram ainda por não incluir um ECAC que comparou os efeitos do ramipril com o telmisartan (ONTARGET), justificando que neste estudo a HTA não fora um requisito de inclusão dos participantes. ${ }^{25}$

Tanto a NOC European Society of Cardiology/European Association for the Study of Diabetes (ESC/EASD), ${ }^{26} \mathrm{de}$ 2013, como a da American Diabetes Association (ADA), ${ }^{1}$ de 2014, recomendaram o uso de um bloqueador do 


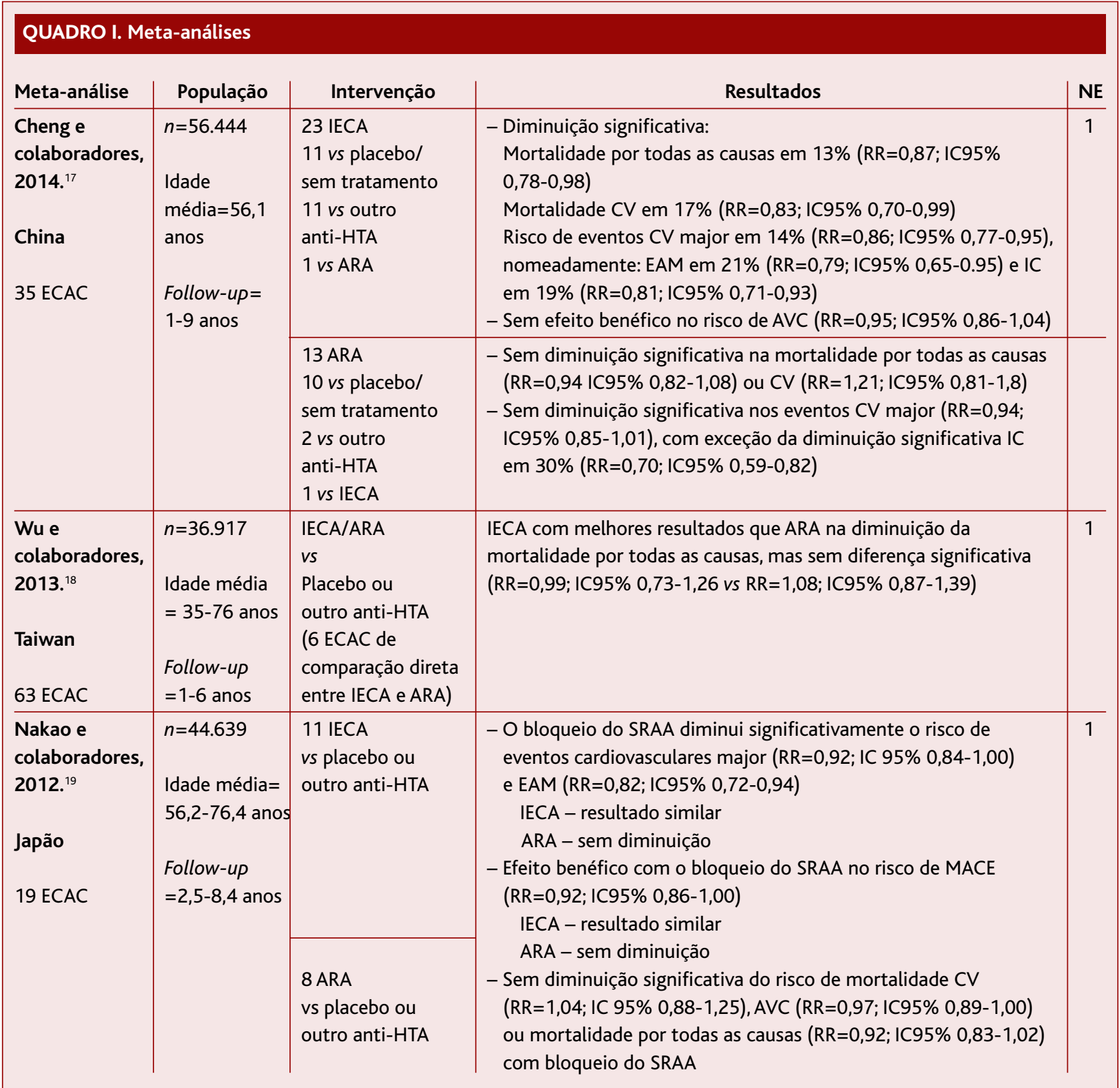

Abreviaturas: AVC - Acidente Vascular Cerebral; ARA - Antagonistas de Recetores da Angiotensina; BCC - Bloqueador dos canais de cálcio; CV - Cardiovascular; EAM - Enfarte Agudo do Miocárdio; ECAC - Ensaio Clínico Aleatorizado e Controlado; IC95\% - Intervalo de Confiança de 95\%; IC - Insuficiência Cardíaca; IECA - Inibidores da Enzima Conversora da Angiotensina; MACE - Major Adverse Cardiovascular Event; NE - Nível de Evidência; RR - Risco Relativo; SRAA - Sistema Renina Angiotensina Aldosterona; VS - versus.

SRAA (IECA ou ARA) no tratamento da hipertensão em diabéticos, não emitindo preferência por nenhuma das duas classes. A ADA acrescenta que se uma classe não for tolerada, deverá ser substituída pela outra. Foi atribuída uma FR A à NOC europeia por ser baseada em quatro
ECAC que compararam IECA ou ARA com outras classes farmacológicas. Os autores da NOC da ADA recomendam os bloqueadores do SRAA relativamente a outras classes, apesar de admitirem que os resultados destes fármacos são conflituosos quando aos efeitos cardiovas- 


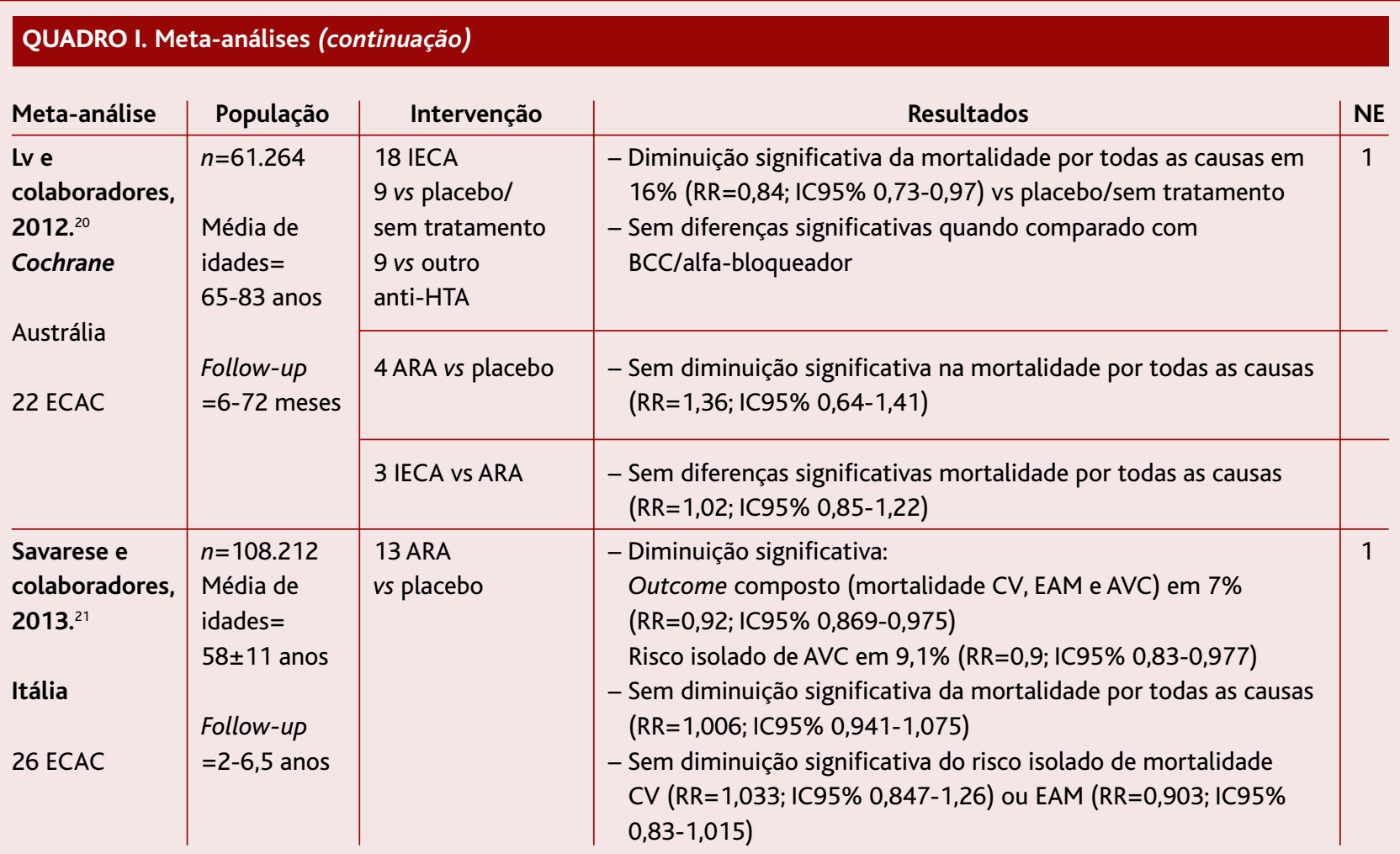

culares. Esta NOC baseia-se na JNC 7, que incluiu estudos entre 1997 e 2003. ${ }^{13}$

As recomendações da Canadian Diabetes Association (2013) advogam o uso de IECA ou ARA em doses que demonstrem proteção vascular nos indivíduos diabéticos hipertensos com doença macrovascular clínica, idade igual ou superior a 55 anos com um fator de risco adicional ou lesão órgão-alvo, idade inferior a 55 anos e complicações microvasculares. ${ }^{27}$ Acrescentam ainda que, para os indivíduos não incluídos nos tópicos anteriores, a terapia inicial apropriada deverá incluir (sem ordem de preferência) IECA, ARA, BCC e diuréticos tiazídicos. Os autores desta NOC suportam as suas recomendações numa MA de 2012, a qual não encontrou diferenças na diminuição de eventos cardiovasculares entre IECA ou ARA. Porém, esta MA incluiu ECAC realizados em pessoas normotensas com ou sem diabetes. ${ }^{28}$

Por último, a Institute for Clinical Systems Improvement (ICSI) recomenda um bloqueador do SRAA no tratamento inicial da hipertensão em DM, afirmando que tanto os IECA com os ARA reduziam a progressão para complicações macrovasculares. ${ }^{29}$ Estas recomendações basearam-se no ECAC HOPE (2000) ${ }^{9}$ e noutro de 2001, realizado por Lewis e colaboradores, apesar deste não ter revelado diferenças nas taxas de mortalidade associadas ao uso de irbesartan comparativamente com amlodipina (houve apenas melhoria para marcadores intermédios renais com o ARA). ${ }^{30}$

As recomendações nacionais da DGS (2013) não foram incluídas na presente revisão, dado que não são evidentes os métodos da sua elaboração nem são percetíveis as bases bibliográficas que as suportam. ${ }^{31}$

\section{CONCLUSÕES}

A evidência disponível foi robusta e consistentemente favoreceu o uso de IECA como primeira opção na prevenção da morbi-mortalidade cardiovascular nesta população (FR A).

Apesar das MA demonstrarem uma diferença do efeito dos IECA e ARA na mortalidade cardiovascular em diabéticos hipertensos, as NOC não emitem uma preferência entre estas duas classes. Os autores da presente revisão desconhecem o motivo pelo qual as MA apresentadas nesta revisão não foram incluídas na elaboração de 


\begin{tabular}{|c|c|c|c|}
\hline Referência & País & Recomendações & $\begin{array}{l}\text { Força de } \\
\text { recomendação }\end{array}$ \\
\hline $\begin{array}{l}\text { ESH/ESC, } \\
2013 .^{4}\end{array}$ & $\begin{array}{l}\text { Vários (UE) } \\
\text { Presidência: } \\
\text { Itália e Bélgica }\end{array}$ & $\begin{array}{l}\text { Todas as classes de agentes anti-hipertensores são recomendadas e podem ser } \\
\text { usadas em doentes com diabetes - individualizar conforme co-morbilidades. } \\
\text { Os bloqueadores do SRAA podem ser preferidos, especialmente na presença de } \\
\text { microalbuminúria ou proteinúria }\end{array}$ & B \\
\hline $\begin{array}{l}\text { JNC 8, } \\
2014 .^{24}\end{array}$ & EUA & $\begin{array}{l}\text { Na população geral (excluindo os de raça negra) e incluindo indivíduos com DM, } \\
\text { o tratamento anti-hipertensivo inicial deve incluir um diurético tiazídico, BCC, } \\
\text { IECA ou ARA } \\
\text { Nos indivíduos de raça negra com DM, o tratamento anti-hipertensivo inicial } \\
\text { deve incluir um diurético tiazídico ou um BCC }\end{array}$ & B \\
\hline $\begin{array}{l}\text { ESC/EASD, } \\
2013 .{ }^{26}\end{array}$ & $\begin{array}{l}\text { Vários (UE) } \\
\text { Presidência: } \\
\text { Suécia e Reino } \\
\text { Unido }\end{array}$ & $\begin{array}{l}\text { No tratamento da hipertensão nos diabéticos recomenda-se um bloqueador do } \\
\text { SRAA (IECA ou ARA), particularmente na presença de microalbuminúria ou de } \\
\text { proteinúria }\end{array}$ & A \\
\hline $\begin{array}{l}\text { ADA, } \\
\text { 2014. }^{1}\end{array}$ & EUA & $\begin{array}{l}\text { A terapia farmacológica nos doentes com DM e HTA deve incluir um IECA ou } \\
\text { ARA } \\
\text { Se uma classe não for tolerada, deverá ser substituída pela outra }\end{array}$ & B \\
\hline \multirow[t]{2}{*}{$\begin{array}{l}\text { CDA, } \\
2013 .{ }^{27}\end{array}$} & Canadá & $\begin{array}{l}\text { Em pacientes com DM e HTA, os IECA ou ARA devem ser a } 1 .{ }^{\text {a linha de }} \\
\text { tratamento }\end{array}$ & \\
\hline & & $\begin{array}{l}\text { Deve ser usado IECA ou ARA, em doses que demonstraram proteção vascular, } \\
\text { para reduzir o risco CV em DM } 1 \text { e } 2 \text { se: } \\
\text { - Doença macrovascular clínica } \\
\text { - Cardiopatia ou nefropatia incluindo microalbuminúria; ou outro fator de risco } \\
\text { (além da DM ou HTA) } \\
\text { - Idade } \geq 55 \text { anos + } 1 \text { fator de risco adicional ou LOA } \\
\text { - Idade < } 55 \text { anos e complicações microvasculares } \\
\text { Para os diabéticos e hipertensos não incluídos nas recomendações anteriores, } \\
\text { uma escolha apropriada inclui (por ordem alfabética): IECA, ARA, BCC e } \\
\text { diuréticos tiazídicos }\end{array}$ & $\begin{array}{l}\text { B } \\
\text { B } \\
\text { B } \\
\text { C } \\
\text { C } \\
\text { B }\end{array}$ \\
\hline $\begin{array}{l}\text { ICSI, } \\
2014.29\end{array}$ & EUA & Os IECA ou ARA devem ser os anti-hipertensores de primeira linha & B \\
\hline
\end{tabular}

Abreviaturas: ADA - American Diabetes Association; ARA - Antagonistas de Recetores da Angiotensina; BCC - Bloqueador dos Canais de Cálcio; CDC - Canadian Diabetes Association; CV - Cardiovascular; ESC/EASD - European Society of Cardiology /European Association for the Study of Diabetes; ESH/ESC - European Society of Hypertension/European Society of Cardiology; EUA - Estados Unidos da América; ICSI - Institute for Clinical Systems Improvement; IECA - Inibidores da enzima conversora da angiotensina; JNC 8 - Eight Joint National Committee; SRAA - Sistema Renina Angiotensina Aldosterona; UE - União Europeia.

nenhuma NOC. Curiosamente, a ADA emitiu novas recomendações em 2016, mantendo o mesmo conteúdo prévio relativamente ao tratamento farmacológico da HTA em indivíduos com DM, bem como do suporte bibliográfico que o sustenta. ${ }^{32}$

Além disso, a maioria das NOC não incluiu os ECAC que estudaram os ARA, que foram maioritariamente publicados após 2005 (nomeadamente ORIENT e ROADMAP). Por outro lado, em várias NOC há falta de clareza na declaração de conflitos de interesse dos autores. $\mathrm{Na}$ NOC da JNC 8, os conflitos de interesse devem ser tidos em conta na sua interpretação, uma vez que vários auto- 
res afirmaram ser consultores e receber honorários de várias empresas de indústria farmacêutica. A NOC do ISCI é clara na inexistência de conflitos de interesse.

Apesar da melhor evidência exposta neste trabalho, há que considerar algumas limitações. Transversal a todas as MA incluídas foi a comparação maioritariamente indireta entre IECA e ARA. Os poucos estudos que compararam diretamente IECA com ARA não demonstraram existir diferenças entre as classes ou demonstraram melhores resultados para os IECA, mas sem diferenças estatisticamente significativas (e.g., DETAIL, 2004). ${ }^{20,33}$ Por outro lado, é de referir a heterogeneidade nos estudos quanto à população, nomeadamente a percentagem de HTA e doença coronária. Além disso, o volume de ECAC que incidiram sobre IECA era ainda frequentemente superior aos ARA. Alguns destes aspetos são claramente identificados pelos autores das MA de Cheng e Wu e seus colaboradores.

Saliente-se a pertinência dos resultados obtidos para a prática clínica, assim como o facto de a pergunta de revisão ter sido orientada para um POEM (Patient Oriented Evidence that Matters). No entanto, é importante relembrar que os resultados desta revisão não poderão ser compreendidos como recomendações.

\section{REFERÊNCIAS BIBLIOGRÁFICAS}

1. American Diabetes Association. Standards of medical care in diabetes, 2014 Diabetes Care. 2014;37 Suppl 1:S14-80.

2. Alvarenga $C$. Hipertensão arterial na diabetes mellitus tipo 2: evidência para a abordagem terapêutica. Rev Port Clin Geral. 2005;21(6):597-604.

3. Gardete-Correia L, Boavida JM, Raposo JF, Mesquita AC, Fona C, Carvalho R, et al. First diabetes prevalence study in Portugal: PREVADIAB study. Diabet Med. 2010;27(8):879-81.

4. Mancia G, Fagard R, Narkiewicz K, Redón J, Zanchetti A, Böhm M, et al. 2013 ESH/ESC Guidelines for the management of arterial hypertension: the Task Force for the management of arterial hypertension of the European Society of Hypertension (ESH) and of the European Society of Cardiology (ESC). J Hypertens. 2013;31(7):1281-357.

5. Giestas, A., et al. Sistema renina-angiotensina-aldosterona e a sua modulação farmacológica [Renin-angiotensin-aldosterone system (RAAS) and its pharmacologic modulation]. Acta Med Port. 2010;23(4):677-88. Portuguese

6. Strippoli GF, Craig M, Deeks JJ, Schena FP, Craig JC. Effects of angiotensin converting enzyme inhibitors and angiotensin II receptor antagonists on mortality and renal outcomes in diabetic nephropathy: systematic review. BMJ. 2004;329:828.

7. Matchar DB, McCrory DC, Orlando LA, Patel MR, Patel UD, Patwardhan MB, et al. Systematic review: comparative effectiveness of angiotensin-converting enzyme inhibitors and angiotensin II receptor blockers for treating essential hypertension. Ann Intern Med. 2008;148(1):16-29.

8. Dézsi CA. Differences in the clinical effects of angiotensin-converting enzyme inhibitors and angiotensin receptor blockers: a critical review of the evidence. Am J Cardiovasc Drugs. 2014;14(3):167-73.

9. Heart Outcomes Prevention Evaluation Study Investigators. Effects of ra- mipril on cardiovascular and microvascular outcomes in people with diabetes mellitus: results of the HOPE study and MICRO-HOPE substudy. Lancet. 2000;355(9200):253-9.

10. Haller H, Ito S, Izzo JL Jr, Januszewicz A, Katayama S, Menne J, et al. Olmesartan for the delay or prevention ofmicroalbuminuria in type 2 diabetes. N Engl J Med. 2011;364(10):907-17.

11. Daiichi Sankyo Co. ORIENT: olmesartan reducing incidence of end stage renal disease in diabetic nephropathy trial - NCT00141453. ClinicalTrials.gov [Internet]; updated 2011 May 9. Available from: https://clinicaltrials.gov/ct2/show/ NCT00141453?term=ORIENT+olmesartan\&rank=1

12. U.S. Food and Drugs Administration. FDA drug safety communication: FDA review of cardiovascular risks for diabetics taking hypertension drug olmesartan not conclusive; label updates required. FDA [Internet]; updated 2016 Jan 1.Available from: http://www.fda.gov/Drugs/DrugSafety/ucm402323.htm

13. Chobanian AV, Bakris GL, Black HR, Cushman WC, Green LA, Izzo JL Jr, et al. The seventh report of the Joint National Committee on Prevention, Detection, Evaluation, and Treatment of High Blood Pressure: the JNC 7 report. JAMA. 2003;289(19):2560-72.

14. Rüster C, Wolf $G$. Renin-angiotensin-aldosterone system and progression of renal disease. J Am Soc Nephrol. 2006;17(11):2985-91.

15. Furtado C. Medicamentos do aparelho cardiovascular: uma análise dos padrões de utilização e despesa em Portugal Continental entre 2000 e 2011 [Internet]. Lisboa: INFARMED; 2012. Available from: http://www.infarmed.pt/portal/page/portal/INFARMED/MONITORIZACAO_DO_MERCADO/OBSERVATORIO/INTRODUCAO_DE_FICHEIROS/Relatorio_ApCardiovascular.pdf

16. Ebell MH, Siwek J, Weiss BD, Woolf SH, Susman J, Ewigman B, et al. Strength of recommendation taxonomy (SORT): a patient-centered approach to grading evidence in the medical literature. Am Fam Physician. 2004;69(3):548-56.

17. Cheng J, Zhang W, Zhang X, Han F, Li X, He X, et al. Effect of angiotensinconverting enzyme inhibitors and angiotensin II receptor blockers on allcause mortality, cardiovascular deaths, and cardiovascular events in patients with diabetes mellitus: a meta-analysis. JAMA Intern Med. 2014; 174(5):773-85.

18. Wu HY, Huang JW, Lin HJ, Liao WC, Peng YS, Hung KY, et al. Comparative effectiveness of renin-angiotensin system blockers and other antihypertensive drugs in patients with diabetes: systematic review and bayesian network meta-analysis. BMJ. 2013;347:f6008.

19. Nakao YM, Teramukai S, Tanaka S, Yasuno S, Fujimoto A, Kasahara M, et al. Effects of renin-angiotensin system blockades on cardiovascular outcomes in patients with diabetes mellitus: a systematic review and meta-analysis. Diabetes Res Clin Pract. 2012;96(1):68-75.

20. Lv J, Perkovic V, Foote CV, Craig ME, Craig JC, Strippoli GF. Antihypertensive agents for preventing diabetic kidney disease. Cochrane Database Syst Rev. 2012;12:CD004136.

21. Savarese G, Costanzo P, Cleland JG, Vassallo E, Ruggiero D, Rosano G, et al. A meta-analysis reporting effects of angiotensin-converting enzyme inhibitors and angiotensin receptor blockers in patients without heart failure. J Am Coll Cardiol. 2013;61(2):131-42.

22. Schmieder RE, Hilgers KF, Schlaich MP, Schmidt BM. Renin-angiotensin system and cardiovascular risk. Lancet. 2007;369(9568):1208-19.

23. Turnbull F, Neal B, Algert C, Chalmers J, Chapman N, Cutler J, et al. Effects of different blood pressure-lowering regimens on major cardiovascular events in individuals with and without diabetes mellitus: results of prospectively designed overviews of randomized trials. Arch Intern Med. 2005;165(12):1410-9.

24. James PA, Oparil S, Carter BL, Cushman WC, Dennison-Himmelfarb C, Han- 
dler J, et al. 2014 Evidence-based guideline for the management of high blood pressure in adults report from the panel members appointed to the Eighth Joint National Committee (JNC 8). JAMA. 2014;311(5):507-20.

25. Yusuf S, Teo KK, Pogue J, Dyal L, Copland I, Schumacher H, et al. Telmisartan, ramipril, or both in patients at high risk for vascular events. $\mathrm{N}$ Engl J Med. 2008;358(15):1547-59.

26. Rydén L, Grant PJ,Anker SD, Berne C, Cosentino F, Danchin N, et al. ESC Guidelines on diabetes, pre-diabetes, and cardiovascular diseases developed in collaboration with the EASD: the Task Force on diabetes, pre-diabetes, and cardiovascular diseases of the European Society of Cardiology (ESC) and developed in collaboration with the European Association for the Study of Diabetes (EASD). Eur Heart J. 2013;34(39):3035-87.

27. Canadian Diabetes Association Clinical Practice Guidelines Expert Committee. Canadian Diabetes Association 2013 clinical practice guidelines for the prevention and management of diabetes in Canada. Can J Diabetes. 2013;37 Suppl 1:S1-212.

28. McAlister FA, Renin Angiotension System Modulator Meta-Analysis Investigators. Angiotensin-converting enzyme inhibitors or angiotensin receptor blockers are beneficial in normotensive atherosclerotic patients: a collaborative meta-analysis of randomized trials. Eur Heart J. 2012;33(4):505-14.

29. Redmon B, Caccamo D, Flavin P, Michels R, Myers C, O'Connor P, et al. Diagnosis and management of type 2 diabetes mellitus in adults [Internet] Bloomington: Institute for Clinical Systems Improvement; 2014. Available from: https://www.guideline.gov/summaries/summary/48544

30. Lewis EJ, Hunsicker LG, Clarke WR, Berl T, Pohl MA, Lewis JB, et al. Renoprotective effect of the angiotensin-receptor antagonist irbesartan in patients with nephropathy dueto type 2 diabetes. N Engl J Med. 2001;345(12):851-60.

31. Direção-Geral da Saúde. Processo assistencial integrado da diabetes mellitus tipo 2: circular normativa ${ }^{\circ}$ 01/2013, de 19/02/2013. Lisboa: DGS; 2013.

32. American Diabetes Association. 2. Classification and diagnosis of diabetes. Diabetes Care. 2016;39 Suppl 1:S13-22.

33. Barnett AH, Bain SC, Bouter P, Karlberg B, Madsbad S, Jervell J, et al. Angiotensin-receptor blockade versus converting-enzyme inhibition in type 2 diabetes and nephropathy. N Engl J Med. 2004;351(19):1952-61.

\section{CONFLITO DE INTERESSES}

Os autores declaram não ter conflitos de interesses.

\section{ENDEREÇO PARA CORRESPONDÊNCIA}

Paula Fernandes Mendes

Rua Dr. José Augusto da Silva, nº 954

E-mail:paulafm3@gmail.com

Recebido em 23-11-2015

Aceite para publicação em 10-09-2016

\section{ABSTRACT}

\section{ANGIOTENSIN CONVERTING ENZYME INHIBITORS OR ANGIOTENSIN RECEPTOR BLOCKERS: EVIDENCE FOR THEIR EFFECTS ON MORTALITY AND MAJOR CARDIOVASCULAR EVENTS IN HYPERTENSIVE DIABETICS}

Objective: Our aim was to assess the evidence for the effects of angiotensin converting enzyme (ACE) inhibitors or angiotensin receptor blockers (ARB) on mortality and major cardiovascular events in individuals with hypertension and diabetes.

Data Sources: National Guideline Clearinghouse, Guidelines Finder, Canadian Medical Association Practice Guidelines, Cochrane Library, DARE, Bandolier, MEDLINE, Index of Portuguese Medical Journals and General Directorate of Health databases.

Methods: The two authors independently performed a search of meta-analyzes (MA), systematic reviews (SR), randomized clinical trials (RCT) and clinical practice guidelines (CPG), published between 31/08/2009 and 31/08/2014 in Portuguese, Spanish, English and French languages. We used the MeSH terms: Diabetes Mellitus AND Hypertension AND Angiotensin-Converting Enzyme Inhibitors OR Angiotensin Receptor Antagonists. The strength of recommendation taxonomy scale was used for the allocation of levels of evidence (LE) and strength of recommendation (SR).

Results: Of the 478 articles found, we included five MA and sixCPG in this report. A Cochrane MA showed that ACE inhibitors significantly decreased mortality from all causes (LE 1) and one MA concluded that this class of medications decreased the risk of myocardial infarction (LE 1). The use of ARB was shown to decrease the risk of stroke in one MA. Another MA showed no significant differences between the two classes of drugs. A recent MA found that ACE inhibitors significantly decreased all-cause mortality, cardiovascular mortality, myocardial infarction, and heart failure (LE 1). In the same MA, ARB decreased the risk of heart failure. Most guidelines recommend ACE inhibitors or ARBs as first line therapy for hypertension in diabetic patients.

Conclusions: The effects of ACE inhibitors and ARB on mortality and cardiovascular events appear to be different. We found consistent evidence favoring the use of ACE inhibitors as a first option in preventing morbidity and mortality in this population (SR A).

Keywords (MeSH terms): Diabetes mellitus; Hypertension; Inhibitors of angiotensin converting enzyme; Angiotensin receptor antagonists. 